\title{
ANALYSIS OF ORIENTATION ACCURACY OF AN UAV IMAGE ACCORDING TO CAMERA CALIBRATION
}

\author{
P. C. Lim, J. Seo, J.Son, T. Kim * \\ Dept. of Geoinformatic Engineering, Inha University, 100 Inharo, Namgu, Incheon, Korea - \\ (vudco88, 12141104, json8520) @inha.edu, tezid@inha.ac.kr*
}

KEY WORDS: UAV, Internal and External orientation, Camera calibration, Inter-model accuracy

\begin{abstract}
:
Utilization of an UAV is increasing because of its easy operation and time saving advantages. Compared with other remote sensing platforms, the biggest difference of a small UAV is the unstable flight attitude due to platform stability. UAVs are equipped with a commercial grade camera, unlike expensive cameras mounted on manned aircraft or satellite platforms. The quality of the map is determined by the characteristics of an UAV and camera performance. In this study, the accuracy of orientation parameters according to UAV camera calibration options was analysed. The camera calibration options were no calibration, self-calibration and calibration by a public calibration toolkit with manual corner measurement. We used four different type of UAVs and three type of SWs. Interior and exterior orientation parameters according to the camera calibration options were obtained from each software. The result of processing by each camera calibration option was different from each other. This may indicate that the UAV camera calibration was not performed accurately and still needed further improvement.
\end{abstract}

\section{INTRODUCTION}

UAVs acquire images at lower altitudes than manned aircraft and can produce higher spatial resolution images. UAV images with high spatial resolution is a pre-requisition for producing maps with high accuracy. For high accuracy maps, however, camera performance is also important. A camera mounted on a UAV is usual a commercial grade camera, which may not be suitable for precise mapping. Therefore, camera calibration is very important and requires thorough verification. Studies on camera calibration and mapping accuracy using an UAV are being actively carried out. The accuracy of the camera calibration was analysed using a smart phone mounted on an UAV (Shin et al., 2016). Other study reported that an inaccurate correction of the image direction and the radial lens distortion of UAV images caused vertical doming errors (James and Robson, 2014).

As the UAV industry develops, various UAVs are also being developed. UAVs are largely divided into fixed-wing and rotary-wing. For the fixed-wing, it is fast and can fly for a long time, and it is possible to shoot a wide area. For the rotary-wing, vertical take-off and landing and free direction control are possible, but flight time is short and speed is slow due to high battery consumption. In recent years, a VTOL (Vertical Take-Off and Landing) that combines these two UAVs characteristics has been released. In this study, we used four different types of UAVs one fixed-wing, two rotary-wing and one VTOL UAVs. In this paper, we tested Pix4DMapper, PhotoScan pro and 3D-UAV for processing UAV images. There are also many commercial software that can process various UAV images. Most of the commercial software includes a camera calibration and bundle adjustment packages.
The aim of this paper is to analyse the orientation accuracy by various camera calibration option using aforementioned UAV image processing software and a public camera calibration toolkit. The focus is mainly on how the camera lens distortion coefficient affects the orientation accuracy. We used three calibration options, which are no-calibration, calibration by the calibration function of the UAV image processing software('self-calibration') and calibration by a public camera calibration toolkit with manual corner measurement('manual-calibration'). In the case of the nocalibration option, processing was performed by setting the lens distortion coefficients to zeros. The self-calibration used the interior lens distortion estimation functions of the UAV image processing software tested. For the manual-calibration, the camera calibration was calculated using the Camera Calibration Toolbox for Matlab for the manual-calibration. The self-calibration was performed automatically by a commercial software. We applied three different types of SWs to four different types of UAVs to calculate a total of 36 orientation accuracy by each calibration option.

\section{EXPERIMENTAL SET-UP}

\subsection{UAV platforms}

As mentioned, we used four different types of UAVs two fixed-wing, one rotary-wing and one VTOL types. For the fixed-wing, Sensefly's eBee with a S.O.D.A camera and SmartPlanes's SmartOne with a Ricoh GR II camera were used. For the rotary-wing, DJI's Inspire2 with a Zemuse X5S camera was used. For the VTOL, Firefly6 Pro from BirdEyeView Aerobotics with a SONY A6000 camera was used.

\footnotetext{
* Corresponding author
} 


\begin{tabular}{|c|c|c|c|c|}
\hline & & \\
&
\end{tabular}

Table 1. The difference types of UAVs

\subsection{Study area}

The study areas were urban and flatland area in Incheon, Korea. We acquired GSD $5 \mathrm{~cm}$ images in two regions. The first region was the city center which is a lot of buildings and loads (Figure 1). We acquired the first region data using eBee, FireFly6 and Inspire2. The second region was the flatland with a playground and some low buildings (Figure 2) We acquired the second region data using SmartOne.

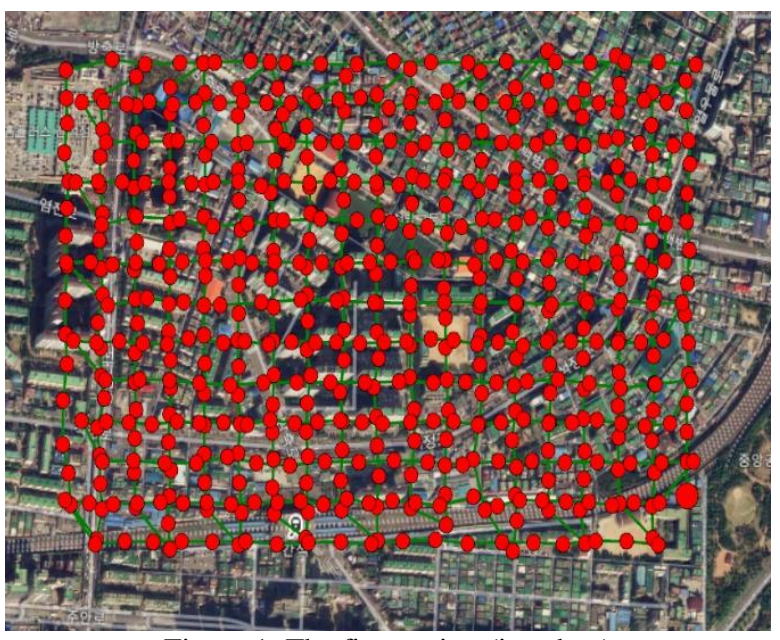

Figure 1. The first region (in urban)

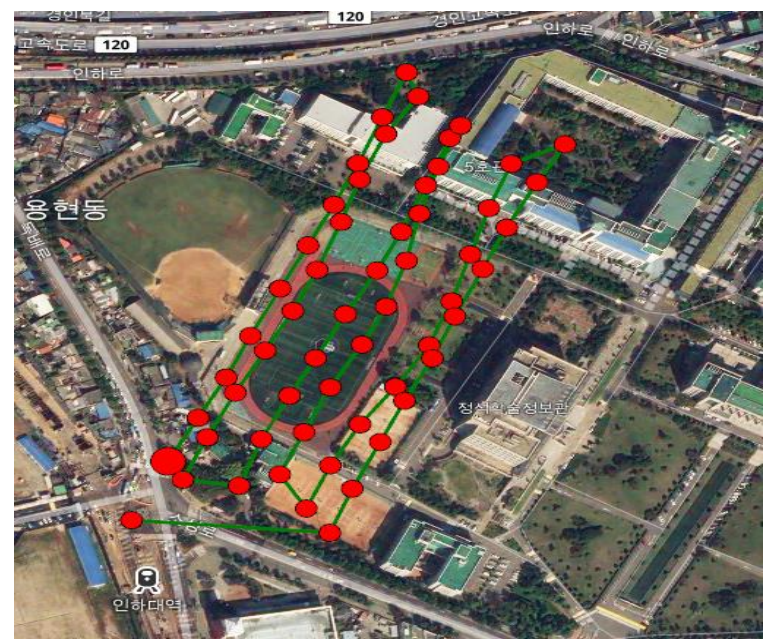

\subsection{Used software}

We used three different types of software Pix4DMapper, PhotoScan and 3D-UAV for processing the UAV images. For the Pix4DMapper, and PhotoScan are well-known software for UAV image processing. 3D-UAV is a software developed internally and an implemented photogrammetric incremental bundle adjustment procedure for precise external orientation parameter estimation. We performed a previous study that compared DSM and height accuracy using 3DUAV and Pix4DMapper (Lim., and Kim., 2018).

\section{METHODOLOGY}

\subsection{Camera calibration for using open software}

In the case of a camera mounted on an UAV, commercial grade camera is mounted unlike an expensive mapping camera mounted on a MAV (Manned Aerial Vehicle). In case of the camera mounted on the MAV, the lens distortion coefficients are close to zero. For a UAV camera, as the distance from the center of image increases, the lens distortion increases. The camera lens distortion coefficients are generally divided into radial $(\mathrm{K} 1, \mathrm{~K} 2, \mathrm{~K} 3)$ and tangential (T1, T2) distortion. The radial distortion is a distortion that occurs as the curvature of the lens moves away from the center of the lens. The tangential distortion is a distortion that occurs when the camera lens and image sensor (CCD, CMOS) were not levelled in the manufacturing process. We performed no-calibration, self-calibration and manualcalibration to analyse the effect of lens distortion of general camera on orientation accuracy. The no-calibration was to set all of the lens distortion coefficients to zeros. For selfcalibration, we set the calibration option in the software and used automatically estimated lens distortion coefficients using only the input images. We used the Camera Calibration Toolbox for Matlab for manual-calibration except SmartOne. In case of Smart One, manual-calibration was not possible due to the absence of the camera. Instead, we used the camera DB value provided by Pix4DMapper for SmartOne. For precise manual-calibration, a chess board was photographed at various angles as much as possible to keep the image from blurring during shooting. The Camera Calibration ToolBox for Matlab allows you to manually set the edge lines of the chess board to get more accurate internal orientation parameter.

Figure 2. The second region (in flatland) 


\section{Establishment of indoor test house}

- Black and white maple chess borad applied - 50 × 40 blackand white patterns

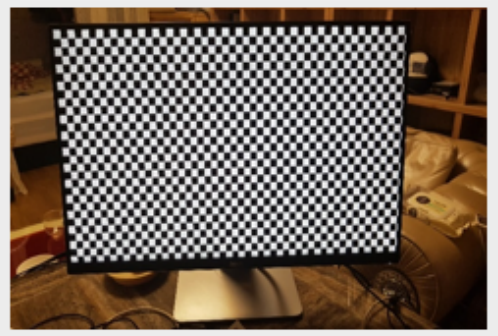

Display on the chess board

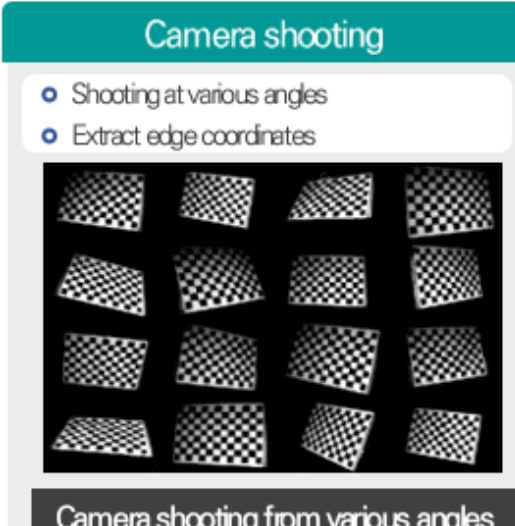

Camera shooting fiom various angles

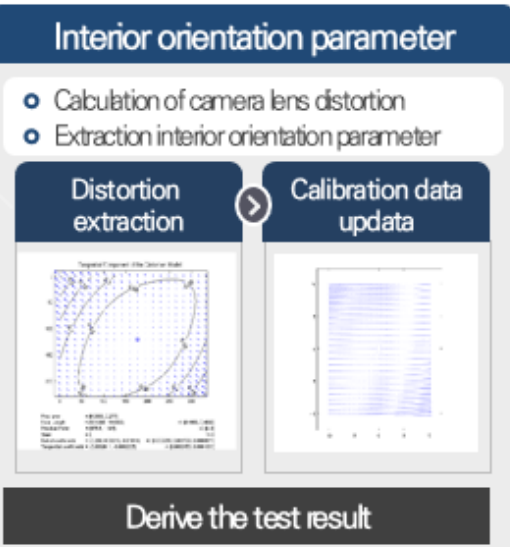

Figure 3. Manual-calibration procedure using Camera Calibration Toolbox for Matlab

3.2 Interior and exterior orientation parameters estimation

The interior orientation parameters were calculated using commercial software and open software as described in section 3.1. For self-calibration, Pix4DMapper, PhotoScan and 3D-UAV use the Brown's distortion model (Brown, 1971) to estimate the camera's interior orientation parameters. We estimated the radial distortion coefficients in each software using images from the urban area as input data (Table 2). In the eBee and Firefly6 data, we confirmed that the distortion coefficients of Pix4DMapper and PhotoScan were almost the same. For the 3D-UAV, the coefficient values were similar but the sign was opposite. This was due to the different definition of the coordinate system.

The exterior orientation parameters were calculated through the bundle adjustment process in each software. For the Pix4DMapper, PhotoScan and 3D-UAV used in this study, tie-points ware created from the input images and exterior orientation parameters were calculated through bundle adjustment.

\subsection{Orientation accuracy analysis}

We developed an analysis procedure which can predict the orientation parameters accuracy as in the Figure 5. First, we generate initial tie-points automatically from UAV images. For updating with undistorted tie-points, we collected these initial tie-points by applying the lens distortion parameters processed each calibration option. Lastly, epipolar geometry was established by applying the exterior orientation parameters and Y-parallex at the undistorted tie-point locations were calculated.

\begin{tabular}{|c|c|c|c|c|c|c|c|c|c|}
\hline \multirow[b]{2}{*}{ Type } & \multicolumn{3}{|c|}{ Pix4DMapper } & \multicolumn{3}{|c|}{ PhotoScan Pro } & \multicolumn{3}{|c|}{ 3D-UAV } \\
\hline & K1 & K2 & K3 & K1 & K2 & K3 & K1 & K2 & K3 \\
\hline eBee & -0.155 & 0.133 & 0.018 & -0.154 & 0.128 & 0.025 & 0.128 & -0.009 & 0.266 \\
\hline Firefly6 & 0.036 & -0.219 & 0.320 & 0.035 & -0.213 & 0.312 & -0.054 & 0.327 & -0.529 \\
\hline Inspire2 & -0.015 & 0.068 & -0.084 & -0.001 & -0.001 & 0.002 & 0.026 & 0.080 & -0.162 \\
\hline
\end{tabular}

Table 2. The calculated radial distortion coefficients in each self-calibration
Inter-model accuracy can be calculated by the difference of object coordinates at tie-points observable in several stereo models. By comparing the $\mathrm{X}, \mathrm{Y}, \mathrm{Z}$ of one stereo model with the same coordinate of $X^{\prime \prime}, Y^{\prime \prime}, Z^{\prime \prime}$ of the other stereo model as show Figure 4, we can predict the inter-model accuracy. The difference between a coordinate accuracy of one (X, Y, $\mathrm{Z}$ ) and other (X", Y", Z") stereo model, where the common point is visible, is ideally zero, but the horizontal and height errors actually occur. In our experiments, we checked the accuracy in several stereo models. We selected multiple images that show common tie-points. 3D-coordinates for the common points were calculated in each stereo pair and their differences were analysed.

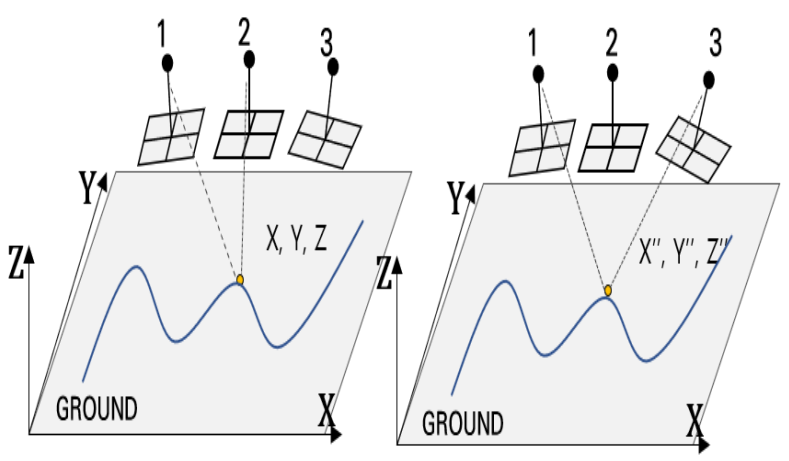

Figure 4. Stereo models with common point 


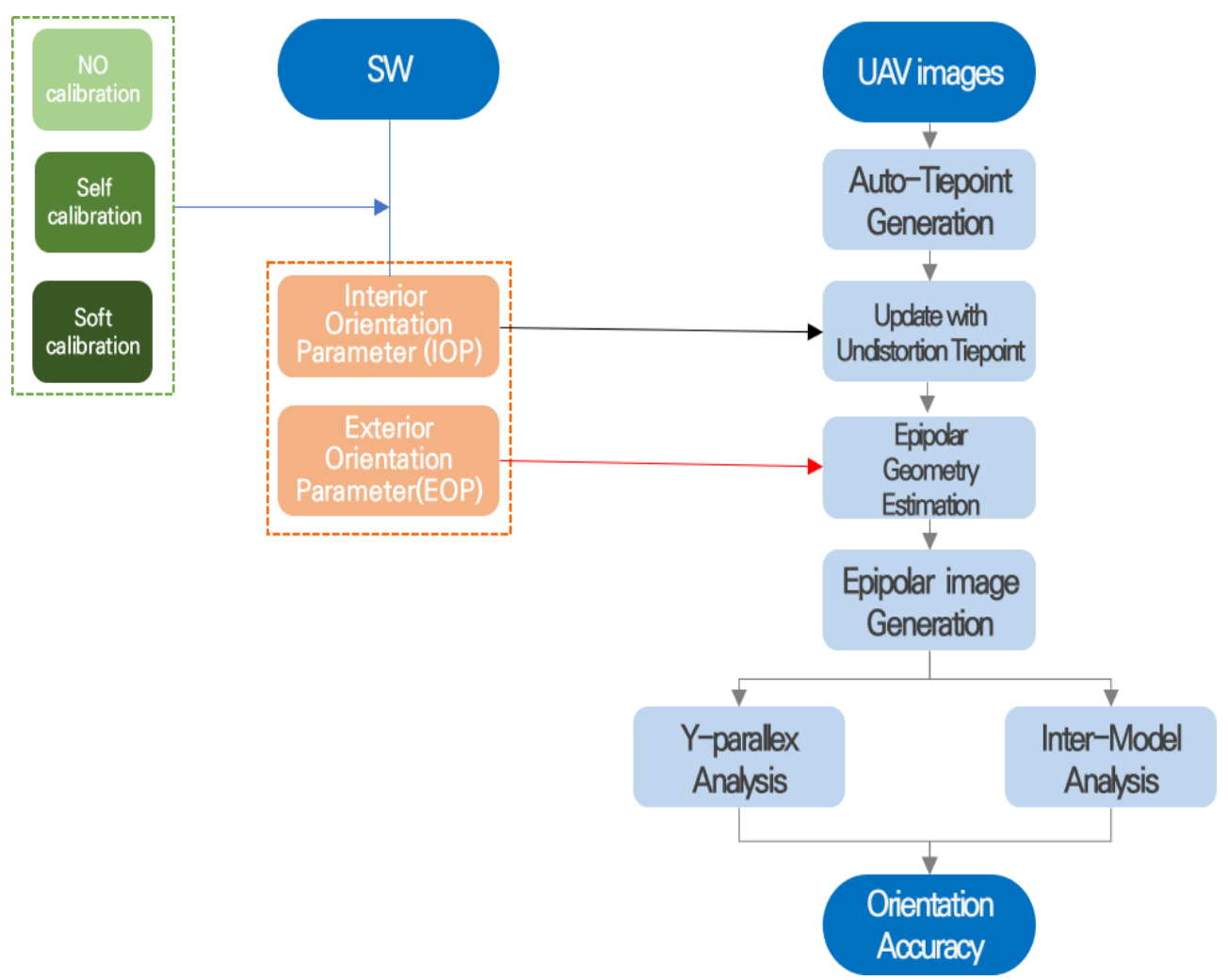

Figure 5. Analysis software process for orientation accuracy

\section{RESULT AND DISCUSSION}

\subsection{Calibration results using Camera Calibration Toolbox for Matlab}

Calibration results as shown in Table 3 were calculated using Camera Calibration Toolbox for Matlab. dPPx and dPPy denote the difference between the lens center coordinate and the principle point coordinate. In the radial and tangential distortion graph, as the radius increases, the radial and tangential distance increase all of cameras. These distorted graphs showed normal distortion characteristics. The radial distortion coefficients were calculated only up to K1 and K2. Tangential distortion is a distortion that occurs when the center of the lens and the sensor do not match in the camera manufacturing process. This distortion is generally negligible. Even with this calibration result, the tangential distortion was close to zero.

\subsection{Orientation accuracy results}

We calculated Y-parallex and inter-model accuracy for a total of 36 data sets (4 UAVs * $3 \mathrm{SWs} * 3$ options). Among them, Y-parallex results were selected only the largest and smallest by each UAV as shown in Table 4 . The smaller the value of Y-parallex and inter-model accuracy, the better the orientation accuracy. If Y-parallex was small, the inter-model accuracy became small. Y-parallex of photoScan's selfcalibration was the largest at Firefly6 only, and Y-parallex of Pix4DMapper's self-calibration was the largest at the other UAVs. In all UAVs, 3D-UAV's Y-parallex was calculated to be the smallest by various calibration options. On the other hand, all calibration options with the largest Y-parallex were self-calibration. From the experimental results, the first reason was that the camera calibration method of selfcalibration was unstable to be applied to UAV. For the second reason, it was judged that the calibration method using the chess board at a short distance may be not suitable for UAV. It was considered necessary to construct a camera calibration environment for UAV.

\section{CONCLUSIONS}

In this study, we analysed the orientation accuracy according to the camera calibration option. Using various UAVs and softwares, we obtained various results. The self-calibration performed by the UAV software was not suitable for UAV. Therefore, manual-calibration was required. We were able to compute Y-parallex and inter-model accuracy using the interior and exterior orientation parameters processed by each software for each calibration option. Although we expected the best results for manual-calibration, Y-parallex and intermodel accuracy according to calibration option were irregular. This implied that calibration of UAV cameras is not working properly. Conventional manual-calibration method used a chess board at close range at various angles. This method may be not suitable for UAVs that acquire images over $100 \mathrm{~m}$ A new calibration environment for camera calibration suitable for UAV is needed. In the future, we plan to build an environment for UAV calibration and analyse the orientation accuracy for manual-calibration. 


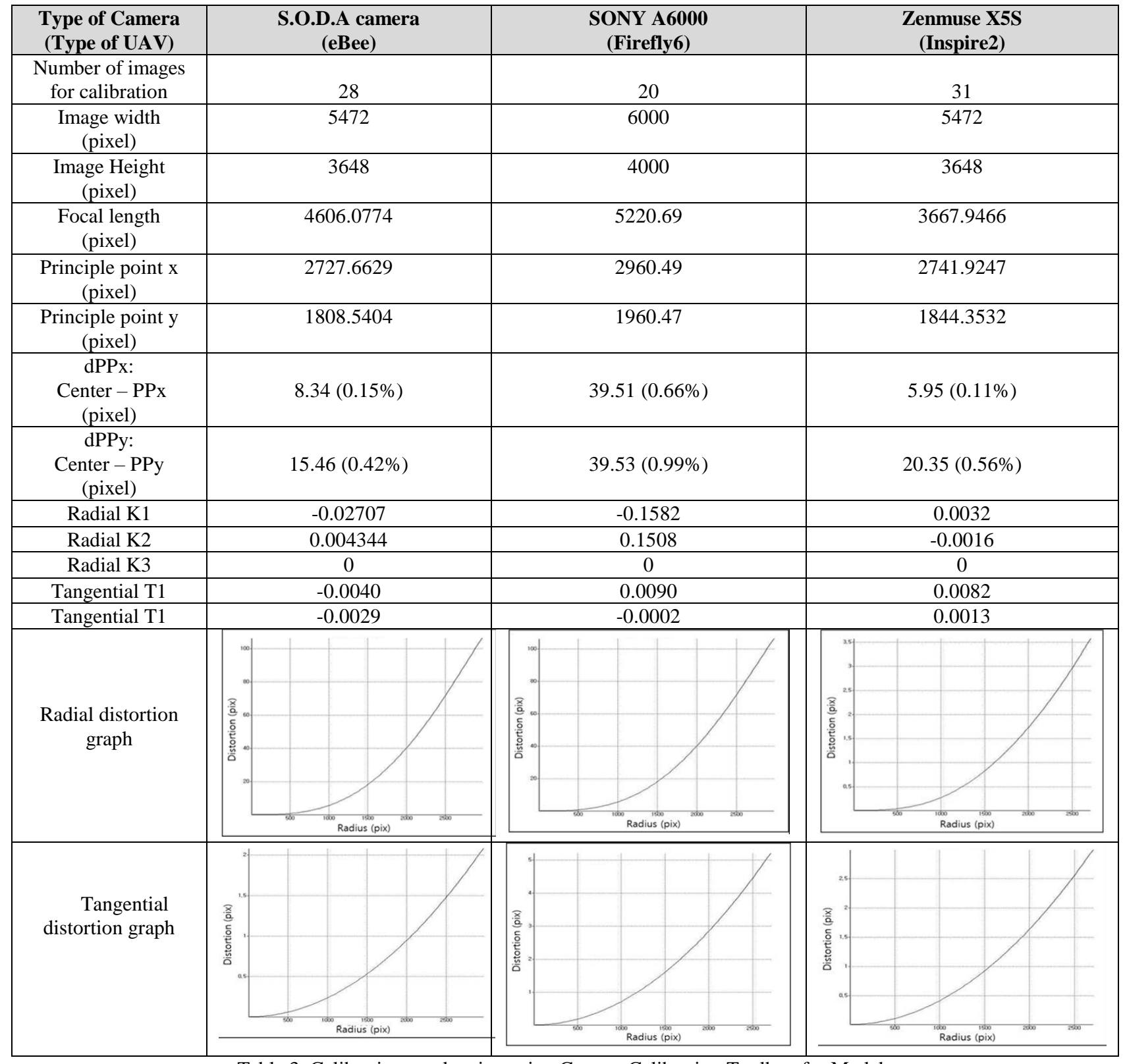

Table 3. Calibration result using using Camera Calibration Toolbox for Matlab

\begin{tabular}{|c|c|c|c|c|c|c|}
\hline \multirow{2}{*}{$\begin{array}{c}\text { Study } \\
\text { area }\end{array}$} & \multirow{2}{*}{ UAV } & \multirow{2}{*}{ SW } & \multirow{2}{*}{$\begin{array}{c}\text { Calibration } \\
\text { option }\end{array}$} & \multirow{2}{*}{$\begin{array}{c}\text { Y-parallex } \\
\text { (pixel) }\end{array}$} & \multicolumn{2}{|c|}{ Inter-Model accuracy (m) } \\
\hline & & & & & $\begin{array}{c}\text { Horizontal } \\
\text { error }\end{array}$ & $\begin{array}{c}\text { Vertical } \\
\text { error }\end{array}$ \\
\hline \multirow{6}{*}{ Urban } & \multirow{2}{*}{ eBee } & Pix4DMapper & self-calibration & 28.1987 & 5.1048 & 11.6357 \\
\hline & & 3D-UAV & self-calibration & 0.8889 & 0.116 & 0.2967 \\
\hline & \multirow{2}{*}{ Firefly6 } & PhotoScan & self-calibration & 9.0674 & 0.9159 & 1.0163 \\
\hline & & 3D-UAV & manual-calibration & 0.9089 & 0.0948 & 0.2547 \\
\hline & \multirow{2}{*}{ Inspire2 } & Pix4DMapper & self-calibration & 13.2448 & 3.3597 & 8.9471 \\
\hline & & 3D-UAV & No-calibration & 0.8517 & 0.1076 & 0.2968 \\
\hline \multirow{2}{*}{$\begin{array}{l}\text { Flat- } \\
\text { land }\end{array}$} & \multirow{2}{*}{ SmartOne } & Pix4DMapper & self-calibration & 22.8024 & 1.3866 & 3.5706 \\
\hline & & 3D-UAV & manual-calibration & 0.7853 & 0.0540 & 0.1509 \\
\hline
\end{tabular}




\section{ACKNOWLEDGENMENTS}

This study was carried out with the support of "Cooperative Research Program for Agriculture Science \& Technology Development (PJ01350003)" Rural Development Administration, Republic of Korea and with the support of "National Geographic Information Institute", Republic of Korea

\section{REFFERENCE}

Shin, D., Han, J., Jin, Y., Park, J., Jeong, H., 2016. Availability Evaluation For Generation Orthoimage Using Photogrammetric UAV System. Korean Journal of Remote Sensing, Vol.32, No3, pp.275-285.

Brown, D., 1997. Close-range camera calibration. Photogrammetric engineering and remote sensing, Vol. 37, No. 8, pp.855-866.

Lim, P.C. and Kim, T., 2018. COMPARATIVE ANALYSIS OF POINT CLOUD GENERATION FROM UAV IMAGES USING VARIOUS COMMERCIAL AND PUBLIC SOFTWARE. 2018 International Symposium on Remote Sensing, Digitally available on USB.

Mike, R.J. and Stuart, R., 2014. Mitigating systematic error in topographic models derived from UAV and ground-based image networks. EARTH SURFACE PROCESSES AND LANDFORMS Earth Surf. Process. Landforms, Vol 39, pp.1413-1420. 